\title{
Negative Pressure Pulmonary Edema- Case Series and Review of Literature
}

\author{
Bhattarai B, Shrestha S
}

Department of Anaesthesiology

\begin{abstract}
Post obstructive pulmonary edema (POPE) also known as Negative pressure pulmonary edema (NPPE)is potentially life threatening complication. It occurs in about $0.1 \%$ of anesthetics and is related to upper airway obstruction. Two types have been described in literature. Different etiology has been attributed to development of Negative pressure pulmonary edema. Early identification and treatment of predisposing factor along with proper monitoring of this complication early treatment should be instituted because resolution is also fast and in most cases without residual effects.
\end{abstract}

\author{
Corresponding Author \\ Basant Bhattarai \\ Department of Anaesthesiology \\ Kathmandu University School of Medical Sciences \\ Dhulikhel Hospital, Kathmandu University Hospital \\ Dhulikhel, Nepal \\ E-mail: basantbhattarai@yahoo.com
}

Citation

Bhattarai B, Shrestha S. Negative Pressure Pulmonary Edema- Case Series and Review of Literature. Kathmandu Univ Med J 2011;36(4):310-4.

\section{KEY WORDS}

negative pressure pulmonary edema, complication, post operative

\section{INTRODUCTION}

Post obstructive pulmonary edema (POPE) also known as Negative pressure pulmonary edema (NPPE) is potentially life threatening complication. It was first demonstrated in 1972 by RL Moore in spontaneously breathing dogs exposed to resistive load. ${ }^{1}$ Although it is an uncommon entity, and occurs in about $0.1 \%$ of anesthetics, it is more often related to upper airway obstruction and in patients who have laryngospasm after procedure under general anesthesia. It is a non cardiogenic pathological process in which there is transudation of fluid into pulmonary interstitium in response to generation of markedly negative intrathoracic pressure. ${ }^{2-4}$ Although different etiologies have been suggested for NPPE, Post extubation stridor and hanging are the most common etiologies.

Here we present three cases of Negative pressure pulmonary edema that we came across in our institute.

\section{CASE ONE}

A 16 yrs old male patient presented with acute appendicitis, for emergency appendicectomy. He had average built and weighed $48 \mathrm{Kgs}$. He had no history of previous anesthetic exposure, there was no history of medical illness. Preoperative investigations were within normal limits except neutrophilic leukocytosis. Chest examination was clear and there were no murmurs on auscultation. Case was accepted as ASA grade $1 \mathrm{E}$. He was shifted to OT with IV line in situ and essential monitors were connected. Rapid sequence induction was done as he was not nil per oral and was posted for emergency surgery. Anaesthesia was induced with thiopental sodium $250 \mathrm{mg}$ and neuromuscular blockade (NMB) achieved with succinylcholine $75 \mathrm{mg}$. He was intubated with $7 \mathrm{~mm}$ cuffed portex endo tracheal tube, after confirming endotracheal tube position in trachea cricoid pressure was released. Inj fentanyl 100 microgram and inj Midazolam 1mg were 
also given. NMB was then continued with vecuronium 4 $\mathrm{mg}$ and intermittent boluses when required. Anesthesia was maintained with Oxygen in Air and Isoflurane 0.6-1\% through closed circuit. Intraoperative vitals were stable and course uneventful. At the end of surgery, the patient had return of spontaneous breathing, residual NMB was reversed with neostigmine $2.5 \mathrm{mg}$ and atropine $1.2 \mathrm{mg}$. It was approximately 30 mins after the last does of relaxant, reversal was given. The patient had good tidal volume, good motor recovery and was responding to verbal commands. He was then extubated. After few minutes of extubation patient became restless and agitated then developed stridor. Oxygen was administered by tight mask and jaw thrust with positive pressure. This relieved the stridor, but after few minutes saturation was falling and the patient had pink frothy sputum coming out of mouth. The patient was immediately reintubated without relaxant and endotracheal suctioning was done which revealed pink colored frothy sputum. He was then connected to anaesthesia ventilator and ventilation was assisted. This still did not help, then with the diagnosis of negative pressure pulmonary edema he was shifted to ICU and mechanical Ventilation and IV antibiotics were initiated. He was connected to ventilator on pressure control mode with Pressure control of 25 and PEEP of 10 and FIO2 of $60 \%$. With these settings he was generating adequate tidal volumes. Chest $X$ ray revealed features suggestive of pulmonary edema. ABG revealed $\mathrm{Sao} 2$ of 88 and $\mathrm{PaO} 2$ of 76. Mechanical ventilation as initiated and cardiology consultation was also done. ECG was normal and bedside echocardiography did not show any abnormality. He was ventilated overnight and serial $x$ ray next morning showed clear lung fields. Endotracheal suctioning was dry. The patient was fully conscious and then was extubated and kept in ICU for observation. He was transferred to ward on day two. He had uneventful recovery then after and was discharged home. The clinical outcome was documented in the case sheet. Here the NPPE could have been attributed to the stridor post extubation.

\section{CASE TWO}

A 35 yrs old patient with humerus fracture was scheduled for plating of humerus under general anesthesia. He had no past medical history and no history of previous anesthesia exposure. He was a snorer. General physical examination showed short neck with no other significant findings. Vital parameters were normal. Chest had bilateral equal air entry and cardiovascular examination was normal. Preoperative investigations were normal. Chest $X$ ray and ECG were within normal limits. The patient was accepted for anesthesia as ASA I physical status and was premedicated with oral lorazepam $2 \mathrm{mg}$ at night and $1 \mathrm{mg}$ in the morning. The surgery was scheduled at $10 \mathrm{AM}$. The patient was shifted to OR and IV line secured. Preoperative monitors connected and premedicated with 100 microgram of fentanyl and $2 \mathrm{mg}$ of Midazolam. After adequate preoxygenation anesthesia was induced with titrated dose of propofol. NMB was achieved with vecuronum $6 \mathrm{mg} / \mathrm{kg}$. After 3 mins of assisted-controlled ventilation, the trachea was intubated with 8.5 sized cuffed portex endotracheal tube. Anesthesia was maintained in usual sequence with Isoflurane in Air and Oxygen with controlled ventilation through closed circuit. Intraoperative vitals were stable and there were no complications of surgery. NMB was maintained with vecuronium appropriate bolus. At the end of surgery, inhalation anesthetics were stopped and after return of spontaneous ventilation residual NMB was reversed with neostigmine and atropine, he was extubated uneventfully and was shifted to post operative recovery unit. He was fully conscious and then he was shifted to post operative ward. Three hours post operatively, had complain of pain for which fentanyl 50 microgram was given after which patient was sedated. Few minutes after that he had difficulty in breathing, he had frothing and froth contained blood tinted sputum. Call was attended for the same. He was given high flow oxygen and saturation was not still maintained. He was shifted to ICU, there he was suspected to have NPPE and was intubated and ET suctioning revealed blood tinted sputum. ABG was done it reveled low $\mathrm{SaO} 2$ and $\mathrm{PaO} 2$ Chest $\mathrm{x}$ ray revealed features suggestive of pulmonary edema. He was connected to ventilator on pressure controlled ventilation with peep of 12 and pressure support of 15 and pressure control of 25 and $\mathrm{FiO} 2$ of 100 . FiO2 was de escalated based on peripheral oxygen saturation. He was investigated for other causes of pulmonary edema including ECG, cardiac enzymes, and echocardiography, which were all normal. He was thus diagnosed as NPPE and ventilated overnight. Next day his chest had improved, still there were signs of pulmonary edema, Fio2 was de escalated to $40 \%$ and PEEP decreased to 8 . On day 2 his chest had improved completely and then he was kept on CPAP with PS of 15 and PEEP of 5. He was then extubated on the same day. He was kept in ICU for Observation for one more day and was then shifted to ward. He had uneventful recovery then after and was discharged home after 10 day after removing the sutures.

\section{CASE THREE}

Twenty yrs old female landed up in Emergency room with the history of hanging. She was brought by neighbors who found her hanging with shawl in the neck. When in the ER, she was not responding well. She was restless and was having red froth from the mouth. There was clear evidence of attempted suicide with mark on her neck. She was immediately intubated and then manual bagging was done with manual resuscitator then was shifted to ICU. She was then connected to ventilator with pressure control ventilation, with peep of 8 and pressure control of 25.FIO2 was 100 on starting but was gradually deescalated based on peripheral Oxygen saturation. Her blood pressure was 70/40 so she was started on dopamine, titrated to the target systolic BP of 90 . She also had bradycardia with 
hert rate fluctuation of 45 to 65 . (Tachybrady arrhythmia) because of attempted suicide. Chest $X$ ray was also done and it revealed pulmonary edema.

Chest improved dramatically the next day crepitation disappeared and it was then clear. Dopamine was slowly deescalated but BP again dropped. USG neck was done it revealed injury around the great vessels. Provisional diagnosis of carotid body injury was made. She was then kept on CPAP with PEEP of 5 and PS of 10 on day 2 then was extubated. Psychiatrist consultation was made and she was kept in observation in ICU for two more days. Dopamine was gradually withheld. She had gradual recover from her carotid body injury too. She was then shifted to Medical ward then was discharged uneventfully after few days.

\section{DISCUSSION}

Postobstructive pulmonary edema (POPE) also referred to as negative pressure pulmonary edema (NPPE) is a potentially life threatening clinical scenario in which immediate-onset pulmonary edema develops after upper airway obstruction. There are two types of NPPE. Type 1 develops after sudden and intense obstruction such as post extubation laryngospasm and epiglottitis; and type 2 , which develops after surgical release of chronic airway obstruction, such as resection of laryngeal tumor, adenoids/ tonsils or intrathoracic goiters. In the adult population, POPE is most commonly caused by laryngospasm and upper airway tumors, whereas in the pediatric age group, epiglottitis, croup, and laryngotracheobronchitis are more common etiologies. ${ }^{3}$

Hanging is one of the commonly used methods of suicide. Death usually occurs in few minutes of hanging form suicide. Pulmonary edema is one of the most common complications that occurs in patients immediately following their rescue from acute airway obstruction or suicidal hanging. ${ }^{2-5}$

As early as 1972, an association between airway obstruction and development of pulmonary edema was suggested in spontaneously breathing dogs exposed to inspiratory resistive load. ${ }^{1}$ The first report of pulmonary edema did not appear till $1973 .{ }^{1}$ First clinical report of the clinical significance of this mechanism was published in $1977 . .^{6}$ The incidence of pulmonary edema associated with airway obstruction is not exactly known, however, the incidence is estimated at $12 \%$ and $11 \%$ in pediatric and adult population requiring active airway intervention for acute upper airway obstruction of various aetiology. ${ }^{2,7}$ Although the incidence is said to be 0.05 to $0.1 \%$ of all anesthetic practices however it is suggested that it occurs more commonly than its generally documented. ${ }^{8}$ The morbidity and mortality associated with under recognized NPPE is as high as $40 \% .{ }^{9}$ A group of patients, especially those with short neck, difficulty in intubation, endotracheal tube obstruction, history of obstructive sleep apnea, obesity, acromegaly and upper aero-digestive tract surgery may have increased susceptibility to this condition. ${ }^{10}$ The frequency of this complication depends upon the type of surgery with greater incidence in ENT procedures. ${ }^{4}$

The principal pathophysiological mechanism involved in the formation of this type of edema is the generation of markedly negative intrathoracic pressure, against a closed glottis (Modified Müller's Maneuver) resulting in markedly negative pleural pressure. Acute respiratory failure also causes release of catecholamine which causes increased blood volume in the lung caused by translocation of blood form the systemic circulation to pulmonary circulation. ${ }^{11}$

Another factor involved in the genesis of this type of acute pulmonary edema is the absence of alveolar ventilation during the period of obstruction causing hypoxemia and hypercapnia, which is associated with anxiety of the patient, lead to release of catecholamine. This hyperadrenergic state causes an increase in left ventricular afterload. Thus reduction in left ventricular compliancy due to deviation of the interventricular septum and the increase in the afterload lead to a reduction in LV ejection volume causing an increase in both ventricular end systolic and end diastolic volumes. ${ }^{12}$

These changes lead to the translocation of the blood form the systemic to low compliance pulmonary circulation resulting in an increase in pulmonary blood volume and micro vascular hydrostatic pressure and consequent fluid transudation from the pulmonary capillaries. This is worsened by pulmonary vasoconstriction induced by hypoxemia and by the reduction perivascular interstitial pressure secondary to pressure gradient. This becomes increasingly more severe due to sustained reduction in Left Ventricular ejection volume resulting in an increase in pulmonary blood volume and pressure. The low complacency of the area contributes to increase in capillary permeability and extravasation of blood cells. This phenomenon was described by West in 1991 as "stress failure." ${ }^{14}$ All these mechanisms add on to transudation of fluid from pulmonary capillary space to the interstitum which progresses to the formation of pulmonary edema. ${ }^{11,15}$ This is due to the increase in systemic arterial pressure resulting from the vasoconstriction caused by the availability if circulating amines, as well as to the increase in the venous return due to reduction $f$ the right arterial pressure secondary to the transmission of the intrathoracic pressure. $^{16}$

Classically, pulmonary edema due to negative intrapleural pressure is accompanied by extravasation of cells from the capillaries secondary to the mechanical failure of alveolar capillary membrane, with consequent alveolar edema and hemorrhage. ${ }^{17}$ Rupture of alveolar capillary membrane might also be involved in NPPE, causing an increase in capillary permeability as well as pulmonary edema. ${ }^{18}$ Diffuse alveolar hemorrhage due to negative intrathoracic pressure is an extremely rare phenomenon. ${ }^{19}$ Pulmonary 
capillary damage caused by the stretching force, followed by mechanical rupture of the alveolar capillary membrane a condition characteristic of diffuse alveolar lesion, has been postulated as the causal factor - a factor that can be potentiated by the rapid change in the transmural pressure, regardless of pressure peaks..$^{20}$

The symptom of NPPE usually develops immediately after extubation though sometimes the onset may be considerably delayed up to few hours in the post operative period. A possible expansion of this delayed manifestation is a positive pressure created by forceful expiration against closed glottis, opposing fluid transudation. ${ }^{21}$ As airway obstruction relieves, increased venous return causes blood shift from peripheral to central circulation and hydrostatic transudation. Thus close post operative observation must be continued for an extended times in patients experiencing respiratory difficulty. This is similar to our case no two who had NPPE few hours after extubation.

Capillary transudation of the proteinaceous material can be part of the classical description of rose colored or foaming serosanguineous discharge in NPPE, in which the extreme elevations in transmural pressure disrupt the alveolar capillary membrane, leading to transudation of erythrocytes to the alveoli and causing frank hemorrhage. ${ }^{22}$

Clinically this condition affects young adults and children, more often with a better prognosis than in older patients. Potential factors for the development of NPPE includes young, strong male patient with short neck, with Mallampatti Grade 3 on physical examination and those with a history of apnea. However none of our patients fitted into these criteria. The higher incidence of this complication in athletes is secondary to the fact that they can develop high negative intrathoracic pressure and maintain a longer period of apnea than other patients. ${ }^{22}$ Mortality and morbidity is increased in patients with lower respiratory reserve and cardiac disease. Under those conditions earlier diagnosis and treatment are important. ${ }^{12}$

Negative pressure pulmonary edema develops rapidly, but its onset can be delayed up to four hours after obstruction has occurred. ${ }^{24}$ Most commonly rapid evolution (in matter of minutes), with improvement in the first 36 hours and resolution with good prognosis, through the use of supplemental oxygen characterize NPPE. However, continuous positive airway pressure is required in 9 to $18 \%$ of cases and 34 to $46 \%$ of patients require controlled mechanical ventilation. ${ }^{25,26}$ The role of fluid restriction and the use of diuretics in this situation are controversial. ${ }^{22}$

Edema accompanied by pulmonary hemorrhage is a rare cause of acute lung injury due to negative pressure in patients with upper airway obstruction. Elimination of the causal factors, together with rapid diagnosis and appropriate treatment, is necessary in order to establish appropriate therapeutic approaches and avoid potentially deleterious iatrogenic complications.
Although controlled mechanical ventilation remains the mainstay of the treatment, use of CPAP or BiPAP through facemask has also been described. ${ }^{27,28}$ Some authors have described eight cases of NPPE treated successfully with Non-invasive positive pressure ventilation. ${ }^{29}$ Once the diagnosis is made aggressive hemodynamic monitoring or vasoactive drugs are not necessary, however the morbidity and mortality can reach up to 11 to $40 \% .^{23}$

\section{CONCLUSION}

Acute pulmonary edema associated with obstruction of the upper airway can aggravate low morbidity surgeries, affecting many young patients. Both surgeons and clinicians must be provided with an understanding that any patient who is otherwise well has the potential to develop Negative Pressure Pulmonary Edema. Extubation of a patient with completely reversed neuromuscular blockade and responsive to simple commands seems to be best form of prevention. When suspected either in post operative period or cases of attempted suicide, early treatment should be instituted because resolution is also fast and in most cases without residual effects.

\section{REFERENCES}

1. Moore RL. The response to respiratory resistance: A comparison of the effects produced by partial obstruction in inspiratory and expiratory phases of respiration. J.Exp Med 1972; 45:1065-80.

2. Oswalt CE; Gater GA; Holmstrom FMG. Pulmonary edema as a complication of acute airway obstruction. JAMA 1997; 238:1833-5.

3. Lang $S A$, Duncan PG, Shephard DA, et al. Pulmonary edema associated with airway obstruction. Can J Anaesth 1990; 37(2):210-8.

4. Tami TA, Lu F; Weldes TO; Kaplan M. Pulmonary edema and upper airway obstruction. Laryngoscope 1986; 86:506-9.

5. Mantha S; Rao SM. Non cardiogenic pulmonary edema after attempted suicide by hanging. Anesthesia 1990;45:993-94.

6. Capitanio MA, Kirkpatrick JA. Obstruction of the upper airway in children as reflected on chest radiograph. PedRadiol 1973; 107:15961.

7. Kanter RK, Watchko JF. Pulmonary edema associated with upper airway obstruction. Am J Dis Child 1984;128:356-8.

8. MC Conkey PP. obstructive pulmonary edema- a case series and review. Anesth Intensive care 2000;28:72-76.

9. Goldenberg JD, Portugal LG, Weingarten RT. Negative pressure pulmonary edema in otolaryngology patient. Otolaryngo Head Neck Surg 1997;117:62-66(s).

10. Hallow KD, Ford EG. Pulmonary edema following post operative laryngospasm: A case report and review of literature. AM Surg 1993;59:443-447(s).

11. Takrouri $M$, Baaj J. Bilateral negative airway pressure pulmonary edema (NPPE) a case report. Middle east journal of anesthesiology 2005;18:631-638.(s).

12. Bisinotto FM, Cardoso Rde P, Abud TM. Acute pulmonary edema associated with obstruction of the airways. Case report. Rev Bras Anestesiol 2008 Mar-Apr;58(2):165-71. 
13. Timby J, Reed C, Zeilender S, Glauser FL. "Mechanical" causes of pulmonary edema. Chest. 1990 Oct;98(4):973-9.Van Kooy MA, Gargiulo RF. Post obstructive pulmonary edema. Am Fam Physician 2000;62(2):401-4.

14. West JB, Tsukimoto K, Mathieu-Costello O, Prediletto R. Stress failure in pulmonary capillaries. J Appl Physiol 1991 Apr;70(4):173142.BBWilms D Shure D. Pulmonary edema due to upper airway obstruction in adults. Chest 1988;94(5):1090-2.

15. Van Kooy MA, Gargiulo RF. Post obstructive pulmonary edema. Am Fam Physician 2000;62(2):401-4.

16. Wilms D, Shure D. Pulmonary edema due to upper airway obstruction in adults. Chest 1988;94(5):1090-2.

17. Dolinski SY, MacGregor DA, Scuderi PE. Pulmonary hemorrhage associated with negative-pressure pulmonary edema. Anesthesiology 2000 Sep;93(3):888-90.

18. Pavlin DJ, Nessky ML, Cheney FW. Increased pulmonary vascular permeability as a cause of re expansion edema in rabbits. Am Rev Respir Dis 1981;124(4):422-7.

19. Schwartz DR, Maroo A, Malhotra A, Kesselman H. Negative pressure pulmonary hemorrhage. Chest 1999 Apr;115(4):1194-7.

20. West JB, Mathieu-Costello O. Stress failure of pulmonary capillaries: role in lung and heart disease. Lancet 1992 Sep 26;340(8822):762-7.

21. Westreich R, Sampson I, Shaari CM, Lawson W. Negative pressure pulmonary edema after routine septorhinoplasty Discussion of pathophysiology, treatment and prevention. Arch Facial PlastSurg 2006;8:8-15(S).
22. Koh MS, Hus AA Eng p. Negative pressure pulmonary edema in the medical intensive car unit. Intensive care Med 2003;29(9):1601-4.

23. Westreich R, Sampson I, Shaari CM, Lawson W. Negative-pressure pulmonary edema after routine septorhinoplasty: discussion of pathophysiology, treatment, and prevention. Arch Facial Plast Surg 2006 Jan-Feb;8(1):8-15.

24. Tarrac SE. Negative pressure pulmonary edema--a postanesthesia emergency. J PerianesthNurs 2003 Oct;18(5):317-23.

25. Rocker GM, Mackenzie MG, Williams B, Logan PM. Noninvasive positive pressure ventilation: successful outcome in patients with acute lung injury/ARDS. Chest 1999 Jan;115(1):173-7.

26. Butterell H, Riley RH. Life-threatening pulmonary oedema secondary to tracheal compression. Anaesth Intensive Care 2002 Dec;30(6):804-6.

27. Marini JJ. Positive end-expiratory pressure in severe airflow obstruction: more than a "one-trick pony"? Crit Care Med 2005 Jul;33(7):1652-3.

28. Antonelli M, Conti G, Moro ML, Esquinas A, Gonzalez-Diaz G, et al. Predictors of failure of noninvasive positive pressure ventilation in patients with acute hypoxemic respiratory failure: a multi-center study. Intensive Care Med 2001 Nov;27(11):1718-28.

29. FuruichiM,Takeda S, Takeda S, Akada $S$ et al. Non invasive positive pressure ventilation in patients with perioperative negative pressure pulmonary edema. J Anesth 2010;24 464-8 